\title{
Central termoeléctrica Bocamina: ¿Deferencia o indiferencia a la Administración? (Corte Suprema)
}

\author{
Comentario de Iván Hunter Ampuero
}

Santiago, 15 de junio de 2012.

VISTOS:

Se reproduce la sentencia en alzada, pero se eliminan sus fundamentos tercero a séptimo.

Y se tiene en su lugar y además presente:

PRIMERo: Que para efectuar una ponderación adecuada del recurso, corresponde expresar que la empresa Endesa S.A. es propietaria de la Primera Unidad de la Central Termoeléctrica Bocamina, ubicada en el sector Lo Rojas, comuna de Coronel, aproximadamente a 30 kilómetros de Concepción.

Igualmente es propietaria del proyecto aprobado ambientalmente mediante Estudio de Impacto Ambiental "Ampliación Central Bocamina (Segunda Unidad)", a través de la Resolución Exenta N 206/07, de fecha 2 de agosto de 2007, que corresponde a una central termoeléctrica de $350 \mathrm{MW}$ de potencia, aledaña a la Primera Unidad mencionada, y finalmente también del proyecto "Optimización Central Termoeléctrica Bocamina Segunda Unidad".

Se expresa que producto de estudios de ingeniería realizados a partir del año 2008, Endesa S.A. ha decidido optimizar el diseño de la Segunda Unidad, circunstancia que le permitiría mejorar su funcionamiento en términos ambientales y de seguridad, sin modificar las características esenciales del proyecto aprobado, lo que, a la vez, podría permitir un aumento de potencia en $20 \mathrm{MW}$. El objetivo general del proyecto es optimizar el funcionamiento de la Segunda Unidad de la CT Bocamina. El predio donde se localiza el proyecto pertenece a Endesa Chile, siendo el acceso a éste por calle Pedro Aguirre Cerda, entre las calles 18 de Septiembre y Mario Fuentealba, frente a la bahía de Coronel. El proyecto se lleva a cabo en las mismas dependencias del actual Complejo Central Termoeléctrica Bocamina; las obras de la Segunda Unidad de la Central Termoeléctrica Bocamina se emplazan en la misma superficie indicada en el proyecto aprobado.

El proyecto "Optimización Central Termoeléctrica Bocamina Segunda Unidad" considera la optimización de la Segunda Unidad de la Central Termoeléctrica Bocamina, a través de modificaciones al diseño de sus obras permanentes. Ello, respecto de las obras 
indicadas en el proyecto "Ampliación Central Bocamina (Segunda Unidad)”, calificado favorablemente mediante la Resolución Exenta N 206/07, de 2 de agosto de 2007, de la Comisión Regional del Medio Ambiente (Corema) de la Región de Biobío, y sus posteriores modificaciones.

Estudios de ingeniería de detalle aludidos concluyeron que se podían implementar mejoras en el diseño original de la Segunda Unidad. Estas mejoras se obtienen a través de la aplicación de un conjunto de modificaciones detalladas más adelante, como por ejemplo: el cambio del tipo de caldera (reemplazando la caldera del tipo "circulación natural" por una caldera del tipo "circulación asistida"), lo que conlleva la redistribución de los equipos y obras de la Segunda Unidad, traduciéndose en un ahorro en los consumos de energía internos y en un aumento posible de $20 \mathrm{MW}$ de potencia. Cada una de estas adecuaciones requiere de otras modificaciones menores, todas necesarias para la operación de esta.

Específicamente, las obras y actividades que se modifican se clasifican en tres grupos, a saber: Primer grupo: obras relacionadas con la optimización de la Segunda Unidad, que corresponden a las modificaciones que son necesarias desde el punto de vista del diseño de ingeniería; Segundo grupo: manejo de insumos y residuos; y Tercer grupo: adecuaciones de seguridad y respaldo, necesarias para asegurar la operación de la Segunda Unidad.

Estos tres grupos de modificaciones tienen efecto en la disposición general de los equipos de la Segunda Unidad (layout).

Las obras modificadas respecto del proyecto aprobado se refieren a lo siguiente:

a.- Obras relacionadas con la optimización de la Segunda Unidad. Con el fin de optimizar la generación de energía, se realizó el cambio del tipo de generador de vapor (caldera), lo cual, a la vez, requiere de la modificación del sistema de refrigeración con agua de mar y del turbogenerador.

b.- Manejo de insumos y residuos.

Carbón: Modificación del sistema de transporte, almacenamiento y manejo en cancha de carbón;

Caliza: Optimización en el sistema de almacenamiento de caliza;

Agua industrial: Modificación de los estanques de almacenamiento de agua industrial;

Petróleo diésel: Modificación de los estanques de almacenamiento de petróleo diésel $\mathrm{N}^{\circ} 2$;

Otros insumos: Modificación en la capacidad de las bodegas de almacenamiento de insumos;

Cenizas: Optimización del sistema de abatimiento de material particulado y sistema de almacenamiento de cenizas volantes y de fondo; $y$

Residuos líquidos: Integración del sistema de tratamiento de Riles.

c.- Otras adecuaciones de seguridad y respaldo. Planta de agua desmineralizada; Transformadores eléctricos; Sistema de generación de aire comprimido; Generador diésel de emergencia; y Medidas de mitigación de ruido.

d.- Obras nuevas respecto del proyecto aprobado. Sistema de colección y tratamiento de aguas lluvias para el sistema de manejo de carbón. 
e.- Obras que se eliminan respecto del proyecto aprobado. Estanque de almacenamiento de petróleo.

Fases del proyecto: Construcción, operación y abandono.

SEgundo: Que en el examen de admisibilidad se deja constancia de que el proyecto "Optimización Central Termoeléctrica Bocamina Segunda Unidad" no contempla ninguno de los efectos, características o circunstancias indicadas en el artículo 11 de la Ley 19.300. Que de otra parte, los antecedentes enunciados dan cuenta fehacientemente que el proyecto "Ampliación Central Bocamina (Segunda Unidad)" fue sometido a Estudio de Impacto Ambiental, mientras que su ampliación tan solo fue presentada a evaluación con una mera Declaración de Impacto por su titular.

Tercero: Que el asunto a dilucidar dice relación a si es suficiente que el proyecto que modifica la generadora "Optimización Central Termoeléctrica (Segunda Unidad)", originalmente evaluado a partir de la presentación de un Estudio de Impacto Ambiental, solo sea sometido en esta oportunidad a Declaración, todo ello a fin de determinar si dicho proceder se encuadra dentro de lo que puede constituir un error evidente, con el objeto de sustituir inmediatamente el procedimiento y de este modo evitar que los interesados deban realizar nuevamente un procedimiento diverso, esto es pasar de Declaración a Estudio.

Cunto: Que en defensa de la suficiencia de la mera Declaración de Impacto Ambiental la recurrida argumenta que el acto que se cuestiona como ilegal y arbitrario constituye un mero examen de admisibilidad y que en tal virtud importa solo el inicio del procedimiento de evaluación ambiental, según establece el artículo 14 ter de la Ley 19.300, siendo suya la opción de hacerlo por medio de Estudio o de Declaración y de la autoridad el control de suficiencia de la opción escogida.

QUINTO: Que no obstante las alegaciones de la recurrida, es un hecho no discutido que la modificación sometida al procedimiento de evaluación de impacto ambiental contempla un conjunto de obras y actividades que tienen efecto en la disposición general de los equipos de la Segunda Unidad, de acuerdo a lo prevenido en la primera consideración, según dan cuenta los antecedentes, todos los cuales inciden de manera importante y determinada en la segunda termoeléctrica a instalar. No solo eso: esos mismos antecedentes informan que las modificaciones apuntan a mejorar su funcionamiento en términos ambientales y de seguridad. Que así las cosas, la calificación de impacto ambiental de la que trata el artículo 11 ter del cuerpo legal anteriormente invocado para los casos de modificación de un proyecto, solo se satisface por medio de un Estudio de Impacto Ambiental y no de una mera Declaración, al contrario de lo que se pretende, pues solo de ese modo es posible establecer si las modificaciones introducidas a la planta generadora importarán el mejoramiento ambiental y no un peligro o daño para el entorno en que se ubica. 
Sexto: La necesidad del Estudio de Impacto Ambiental en este caso resulta abonada por los principios que inspiran y sobre los cuales se desarrolla la regulación ambiental en nuestro ordenamiento, en particular los principios de prevención y de responsabilidad, que solo se cumplen si los evaluadores aplican desde un inicio sus especiales conocimientos sobre la materia, cuestión que solo resulta posible con un Estudio de Impacto Ambiental, al igual como se hizo al momento someter a ese procedimiento los proyectos originales, y no con una mera Declaración de parte interesada como ahora se pretende, que a todas luces es insuficiente.

SÉPTIMO: Que si bien el acto recurrido constituye una decisión administrativa adoptada dentro del ámbito de la competencia del órgano respectivo, la declaración de suficiencia sin embargo envuelve una decisión carente de razonabilidad y por tanto arbitraria, pues no se entiende cómo una Declaración de Impacto Ambiental permita evaluar una cuestión técnica compleja, que dice relación con la modificación de un proyecto de generación de energía eléctrica cuya evaluación primitiva requirió precisamente de un Estudio. Desde el momento que el acto cuestionado no contiene fundamentos ni motivos que den cuenta de las razones consideradas por la autoridad para adoptar tal decisión, lo así resuelto deviene en arbitrario, pues aparece como una actuación desprovista de sustento, más producto de la pura voluntad de su autor que de fundamentos que la expliquen y legitimen, pues un proyecto que fue aprobado por Estudio de impacto ambiental y que ahora pretende modificaciones de la importancia y entidad que ya han sido referidos no puede ser sometido a una mera Declaración.

Octavo: Que en otro orden de cosas, se rechazará la alegación de la recurrida de carecer los recurrentes "Confederación Nacional de Pescadores Artesanales" de legitimación activa, en atención a que reiteradamente la jurisprudencia y la doctrina han sostenido que la legitimación activa en un recurso de protección ha sido reconocida ampliamente a cualquier persona, natural o jurídica, afectada "en sus derechos", no estando nadie excluido de su ejercicio, en especial si se considera que directa e indirectamente pueden verse indudablemente afectados por la obra cuya evaluación ambiental se cuestiona en su procedimiento.

Por estas consideraciones y de conformidad con lo que dispone el artículo 20 de la Constitución Política de la República y el Auto Acordado de esta Corte sobre la materia, se revoca la sentencia de nueve de abril pasado, escrita a fojas 283 , se declara que se acoge el recurso de protección deducido en lo principal de la presentación de fojas 17 y se ordena que el titular del proyecto "Ampliación Central Bocamina (Segunda Unidad)" presente un Estudio de Impacto Ambiental a la Comisión de Evaluación de la Región del Bío Bío.

Regístrese y devuélvase con sus documentos.

Redacción a cargo del Ministro señor Muñoz.

Rol: 3141-2012. 


\section{COMENTARio}

Este reciente fallo confirma la tendencia casi generalizada de judicializar los procedimientos administrativos de evaluación de impacto ambiental. Sin embargo, esta sentencia presenta una serie de aristas que pueden resultar de interesante análisis, tanto desde el derecho ambiental como del administrativo. Comenzaré con lo más básico.

La Corte Suprema (en adelante la Corte) determina que el acto inicial del procedimiento de evaluación ambiental, esto es, el que declara su admisibilidad, es susceptible de vulnerar el derecho fundamental a un medio ambiente libre de contaminación. Se trata de un acto que desde el derecho administrativo se conoce como acto de trámite, que no contiene una decisión final de la Administración sino que colabora con la substanciación normal del procedimiento. Con esto, el paso que da la jurisprudencia es aun mayor al que dio en el caso Campiche ${ }^{1}$ cuando estimó que la resolución de calificación ambiental, al ser un acto terminal, tenía aptitud para generar una vulneración al medio ambiente. Ahora hasta un mero acto de trámite del procedimiento es capaz de infringir garantías constitucionales. El ámbito en que puede extenderse el control jurisdiccional comienza a ocupar espacios que antes estaban reservados exclusivamente a la Administración.

En segundo lugar, y relacionado con lo anterior, es claro que la jurisdicción ha comenzado a adoptar decisiones que corresponden al ámbito administrativo. Este caso es quizá un ejemplo patente. Es cierto que la Corte invoca la falta de motivación o razonamiento del acto de trámite como habilitación para adoptar su decisión, pero también es cierto que la determinación de cuál es el procedimiento que debe seguir un determinado proyecto o actividad es algo que le corresponde privativamente a la Administración. Es razonable exigir un cierto estándar de fundamentación a la Administración en la dictación de sus actos decisionales, ello es además consustancial al ejercicio del poder en un Estado de Derecho $^{2}$. Pero de ello no se sigue que los tribunales puedan adoptar la decisión por la Administración cuando los actos de esta no satisfacen ese estándar ${ }^{3}$. Es de esperar que con la limitación del Art. 26 de la Ley de Tribunales Ambientales se respete aquella

${ }^{1}$ C. Suprema, sentencia de 22 de junio de 2009, Rol No 1219-2009.

${ }^{2}$ Un interesante fallo acerca de la fundamentación de los actos de la administración en materia ambiental es la sentencia de la C. Suprema, de 26 de noviembre de 2012, Rol No 7071-2012, referido a la Declaración de Impacto Ambiental de la Central Termoeléctrica Pirquenes. Esta sentencia confirma la tendencia jurisprudencial incoada, con mayor énfasis, a partir de la Ley $\mathrm{N}^{\circ} 19.880$ sobre procedimientos administrativos, de exigir la explicitación de motivos de los actos de la Administración. Vid., C. Concepción, 18 de octubre de 2005, RDJ17451, MJJ17451; C. Punta Arenas, 16 de abril de 1996, RDJ753, MJJ753, Rol No 7.970; C. Santiago, 4 de julio de 2008, Rol: 8273-07, MJJ17546. En doctrina puede verse: Cordero, Luis, El control de la administración del Estado, p. 166.

${ }^{3}$ El fallo precedentemente citado es un buen ejemplo de esa necesaria deferencia competencial que debe existir entre ambos poderes del Estado. La Corte de Apelaciones de Concepción había dispuesto que el Proyecto Central Termoeléctrica de Pirquenes reingresara al SEIA bajo la modalidad de EIA, y no conforme una Declaración. La Corte Suprema, en cambio, entendió que no podía adoptar una decisión que estaba situada en las competencias administrativas del SEA, por lo que ordenó que la Comisión del Art. 86 de la ley se pronunciara nuevamente sobre la calificación ambiental del proyecto. 
condición de deferencia que debe tener la jurisdicción sobre las competencias asignadas a la Administración, al menos, cuando se trate de actos de contenido discrecional.

Otro aspecto importante es el criterio adoptado por la Corte para decidir que la modificación al proyecto debía someterse a un Estudio de Impacto Ambiental. Como se sabe, el Art. 11 ter de la Ley N 19.300 introducido por la reforma del año 2010, establece que "en caso de modificarse un proyecto o actividad, la calificación ambiental recaerá sobre dicha modificación, y no sobre el proyecto o actividad existente". Al respecto la norma no dice bajo qué procedimiento debe someterse la modificación del proyecto o actividad, cuestión que resulta absolutamente obvia, ya que el instrumento de evaluación dependerá de los impactos que sean susceptibles de producirse.

La decisión de la Corte, no obstante, prescinde completamente de la determinación de si el proyecto o actividad, que constituye la modificación de la Segunda Unidad, es susceptible de producir los impactos que justifican la elaboración de un Estudio de Impacto Ambiental (en adelante EIA). Por el contrario, introduce un criterio -algo dudoso-, como es el de la idoneidad técnica de la Declaración de Impacto Ambiental (en adelante DIA). A su juicio, este instrumento "no permite evaluar una cuestión técnica compleja”. Una decisión como esta, perfectamente posible, corresponde adoptarla en primer término a la Administración y, luego, eventualmente podrá ser revisada por la jurisdicción. Aquí, sin embargo, la Corte evade todo el procedimiento administrativo para adoptar ella misma una decisión. Ni siquiera concurre un supuesto de "sustitución", sino que lisa y llanamente existe una "usurpación" de potestades.

Aunque no se diga expresamente, puede entenderse que la Corte define una exigencia de identidad de los instrumentos de evaluación entre un proyecto y su modificación, de manera que el titular del proyecto que pretenda salirse de esa exigencia deberá justificarlo suficientemente. Hay una suerte de desconfianza a autorizar la modificación de un proyecto en una forma distinta al instrumento con que fue evaluado previamente. Nada garantiza, según la Corte, que las modificaciones que se vayan a efectuar no importen un peligro o daño para el entorno en que se ubica. A mi entender este criterio no parece del todo razonable, debido a que no es posible sustentar que el legislador al disponer que la evaluación recaiga sobre la modificación del proyecto esté exigiendo una identidad de los instrumentos, sin perjuicio de que eventualmente puedan quedar sujetos al mismo. Un criterio que podría ser útil, por representar la aplicación estricta de las normas legales, es determinar si la modificación del proyecto o actividad por sí sola es susceptible de generar los efectos, características o circunstancias del Art. 11, incluyendo obviamente los impactos sinérgicos. 